\title{
Characterizing the Electrical Properties of Anisotropic, 3D-Printed Conductive Sheets
}

\author{
Alexander Dijkshoorn, Martijn Schouten, Gerjan Wolterink, Remco Sanders and Gijs Krijnen \\ University of Twente, Robotics and Mechatronics, Enschede, the Netherlands \\ a.p.dijkshoorn@alumnus.utwente.nl
}

\section{SUMMARY AND MOTIVATION}

This paper introduces characterization techniques to investigate electrical properties of $3 \mathrm{D}$ printed conductors. It presents a physical model to describe frequency dependent electrical properties of 3D-printed conductors; the use of infrared thermography to characterize electrical anisotropy in 3D-printed sheets and the use of the voltage contrast Scanning Electron Microscopy method (VCSEM) to determine potential distributions in 3D-printed sheets. The characterization methods could enable improvement of 3D-printed transducer design and exploit electrical properties of 3D-printed conductors.

\section{ADVANCES OVER PREVIOUS WORKS}

3D-printing conductors, and in particular transducers, by means of fused deposition modelling is an upcoming research area [1], where 3D-printed piezo resistive, EMG and capacitive sensors have been demonstrated [2] and a significant amount of research has been done on electrical properties of conductive-polymer composites for printing $[3,4]$. However, printing conditions remarkably affect the electrical properties due to voids and bonding conditions between adjacent traxels (i.e. track-elements), as shown by measurements and simulations [4,5]. Insight is gained by developing appropriate physical models, representing conductive structures by fused traxels. In previous research conductance was already described in this way; however this was limited to a 1D-solution in the DC-case [6]. Furthermore, electrical characterization has primarily focused on global impedance measurements, whereas the $2 \mathrm{D}$ distribution of the electrical impedance is of interest. Here we show that IR thermography, so far used for studying heating power in 3Dprinted samples [4], can also be used for studying the anisotropic electrical properties of 3D-printed samples as has been done for characterization of carbon fibre reinforced polymers [7]. Next it is shown that VCSEM, used e.g. to characterize conducting networks in CNT composites [8], can also be applied to $3 \mathrm{D}$ printed conductors.

a.

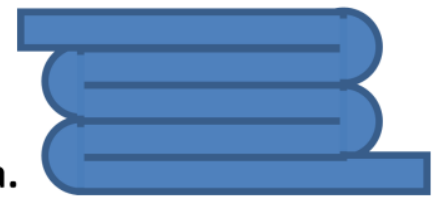

b.

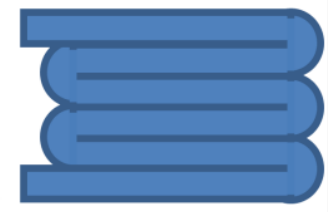

Fig. 1: Printed sample design with leads on opposite (a) and same (parallel) side (b).

\section{METHODOLOGY}

Two different printing designs are used, with leads on the opposite and parallel side (Fig 1). The conductors are simulated by means of traxels with bulk (resistivity $\rho$, relative permittivity $\epsilon_{\mathrm{r}}$ ) and mutual contact properties (inter-traxel resistivity $\sigma$, inter-traxel capacitance $C_{0}$ ) to describe the electrical characteristics, making it an $\mathrm{AC}$-extension of the work by Hampel et al. [6]. An equivalent electrical network to represent these properties is shown in Fig 2.

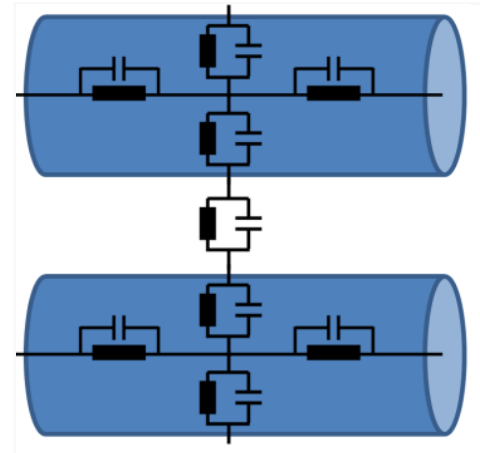

Fig. 2: Physical model showing traxels with an equivalent electrical network representing the $2 \mathrm{D}$ bulk and inter-traxel impedances.
Finite Element Experiments are performed for validation of the FEM simulations with COMSOL software.

CAD designs are sliced with Simplify3D and 3D printed from flexible carbon black-filled TPU (Palmiga Innovations PIETPU 85-700+ [9]) using a Flashforge with Flexion extruder. The samples are single-layer sheets of $15 \mathrm{~mm}$ by $15 \mathrm{~mm}$ printed on glass wafers with electrical contact leads on either the same (parallel) or opposite side (Fig 1), made via copper tape with Ag-conductive paint (Electrolube SCP26G).
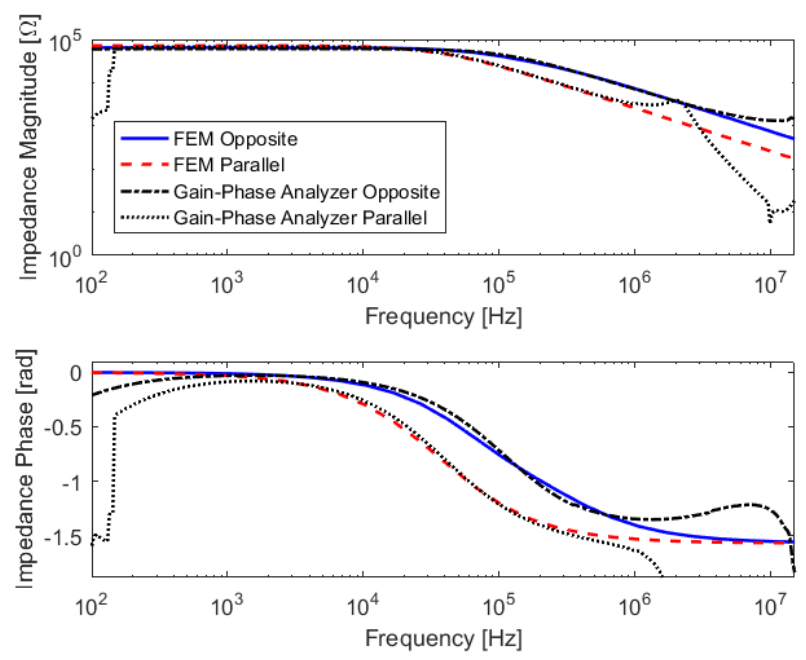

Fig. 3: Impedance and phase data of the FEM simulations in comparison with the gain-phase measurements. 
Impedance measurements are done using a gain-phase analyser (HP 4194A). Since printing conditions influence contact properties, the two dissimilar designs (Fig 1) are fitted separately. DC thermal measurements are based on temperature increases due to resistive heating, using infrared thermography with an IR camera (FLIR ONE Gen 2).

However, actual heat transport may cause differences between simulations and measurements. To have more clear measurements, samples with larger inter-traxel resistivity are used for the thermal measurements.

DC VCSEM measurements are done with an FEI Quanta 450, where the different leads have a different bias relative to the ground of the SEM. A more positive bias reduces the number of secondary electrons reaching the detector, giving rise to a nonlinear dependency [8]. By means of creating a SEM intensity calibration curve for a sample for different voltages, voltage distributions of samples are reconstructed by fitting and inverting in a pixel-wise manner to reduce SEM artefacts.

\section{RESULTS AND DISCUSSION}

Fig 3 shows the gain-phase measurements compared to the FEM simulations showing a good fit from $1 \mathrm{kHz}$ to $1 \mathrm{MHz}$. The fitted parameters are presented in Tab 1. Some deviations occur at both lower (around $100 \mathrm{~Hz}$ ) and higher frequencies (around $1 \mathrm{MHz}$ ) for which the cause is still unclear.

\begin{tabular}{ccc}
\hline Variable $\backslash$ Sample & Opposite & Parallel \\
\hline Resistivity $\rho[\Omega m]$ & 2.8 & 2.8 \\
Relative permittivity $\epsilon_{\mathrm{r}}[-]$ & $0.9 \mathrm{e} 5$ & $1.5 \mathrm{e} 5$ \\
Inter-traxel resistivity $\sigma\left[\Omega \mathrm{m}^{2}\right]$ & $2 \mathrm{e}-3$ & $2 \mathrm{e}-3$ \\
Inter-traxel capacitance $C_{0}\left[\mathrm{~F} / \mathrm{m}^{2}\right]$ & $2.8 \mathrm{e}-4$ & $4 \mathrm{e}-3$ \\
\hline
\end{tabular}

Tab. 1: Fitting parameters for the impedance and VCSEM simulations.

Fig 4 shows the dissipated power density in the FEM simulations compared to measured thermal images with temperatures ranging from $24^{\circ} \mathrm{C}$ to $28^{\circ} \mathrm{C}$. The general power and temperature distributions are similar, with most dissipation in the leads and around the edges. Though the

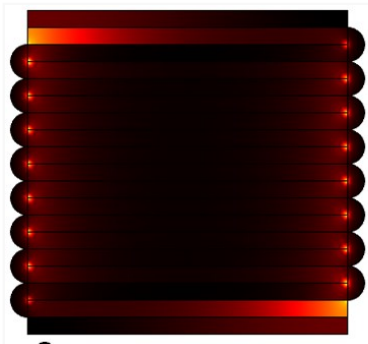

a.

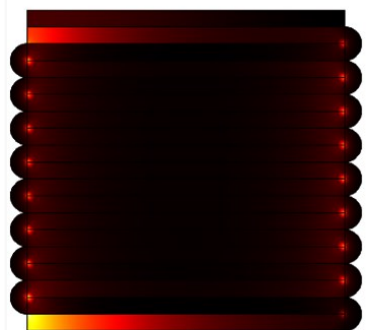

C.

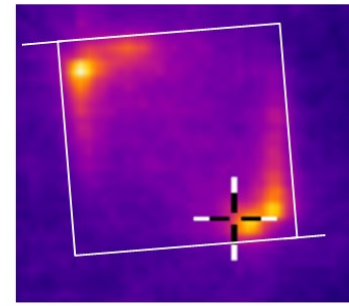

b.

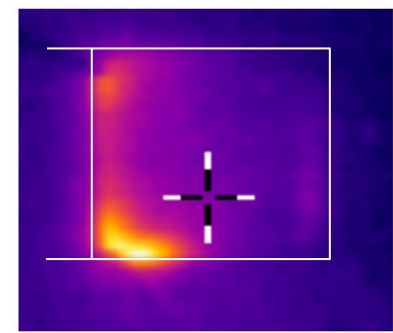

d.
Fig. 4: DC Power density of FEM (a, c) simulations versus thermal measurements (b, d) of the opposite lead (fig. 1.a) and parallel lead sample (fig 1.b).
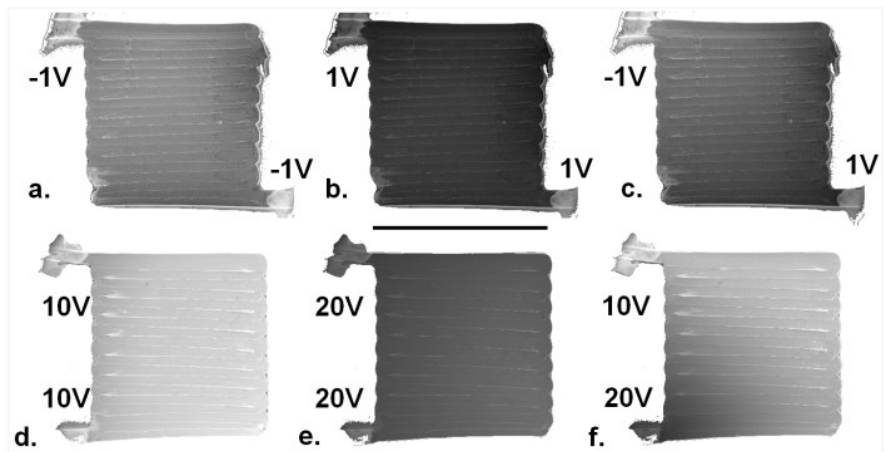

Fig. 5: VCSEM results with constant voltage images for calibration (a, b, d, e) and the contrast of a measured voltage distribution (c, f) of the opposite lead (fig 1.a) and parallel lead sample (fig 1.b). The central bar indicates $15 \mathrm{~mm}$.

measurements have a more spread out distribution, which is expected to be due to thermal conduction.

Fig 5 shows DC VCSEM images as used for pixel-wise calibration (4.a, 4.b, 4.d, 4.e) and as used for voltage distribution analysis (4.c, 4.f). The calibration images have the same contrast everywhere in the samples, apart from the topographic and edge effects of the SEM. The measured voltage distribution images show a clear transition in contrast between both leads.

Fig 6 gives the corresponding FEM simulations and experimental results, showing both qualitatively and quantitatively good correspondence. The results show almost isotropic conduction in the samples, due to a low contact resistance (Tab 1).

In conclusion characterization methods have been presented to predict the electrical properties of 3D-printed conductors in combination with a physical model for FEM simulations. Classical impedance measurements have been used as validation of the FEM simulations. Infrared thermography and voltage contrast SEM have been applied for DC

characterization, showing promising results. Future work will focus on the influence of the printing parameters on the intertraxel properties and on improving the characterization methods and extending them to AC measurements.

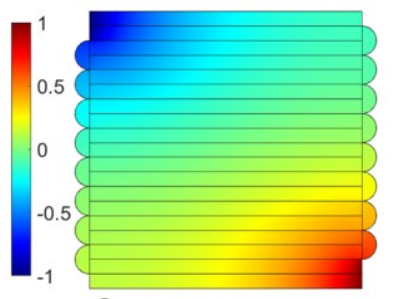

[V]

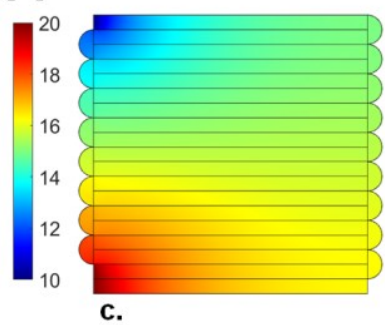

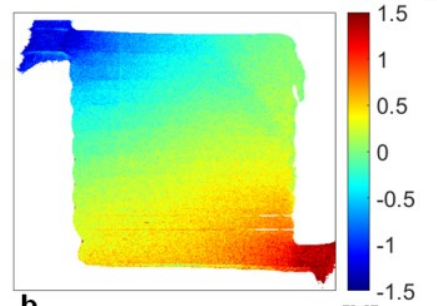

b.
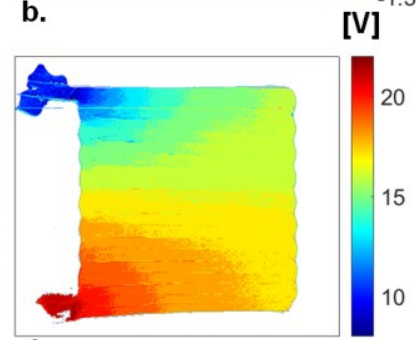

d.
Fig. 6: VCSEM DC voltage distribution of FEM simulations (a,c) compared to the experimental results $(b, d)$. 


\section{REFERENCES}

[1] Xu, Y.; Wu, X.; Guo, X.; Kong, B.; Zhang, M.; Qian, X.; Mi, S.; Sun, W., "The Boom in 3D-Printed Sensor Technology,". Sensors 2017, 17, 1166.

[2] Dijkshoorn, A., Werkman, P., Welleweerd, M., Wolterink, G., Eijking, B., Delamare, J., Sanders, R., and Krijnen, G. J. M.: "Embedded sensing: integrating sensors in 3-D printed structures," J. Sens. Sens. Syst., 7, 2018, pp. 169-181.

[3] Kwok, S.W.; Goh, K.H.H.; Tan, Z.D.; Tan, S.T.M.; Tjiu, W.W.; Soh, J.Y.; Ng, Z.J.G.; Chan, Y.Z.; Hui, H.K.; Goh, K.E.J., "Electrically conductive filament for 3D-printed circuits and sensors," Appl. Mater. Today, 2017, 9, pp. 167-175.

[4] Watschke, H., Hilbig, K., Vietor, T., "Design and Characterization of Electrically Conductive Structures Additively Manufactured by Material Extrusion," Appl. Sci. 2019, 9, 779.
[5] Zhang, J.; Yang, B.; Fu, F.; You, F.; Dong, X.; Dai, M., "Resistivity and Its Anisotropy Characterization of 3D-Printed Acrylonitrile Butadiene Styrene Copolymer (ABS)/Carbon Black (CB) Composites," Appl. Sci. 2017, 7, 20.

[6] Hampel, B., Monshausen, S., Schilling, M., "Properties and applications of electrically conductive thermoplastics for additive manufacturing of sensors," Tech. Mess. 2017, 84, pp. 593-599.

[7] Athanasopoulos N., Kostopoulos V., "A comprehensive study on the equivalent electrical conductivity tensor validity for thin multidirectional carbon fibre reinforced plastics," Composites Part B, 2014, 67, pp. 244255.

[8] Spallek, S., "Electron Microscopy Studies on Structure-property Relationships of Nanoparticulate Transparent Electrodes for Printed Electronics," Ph.D. thesis, 2016, Friedrich-Alexander-Universität, Erlangen-Nürnberg.

[9] Palmiga Innovations, Rubber 3d printing, http://rubber3dprinting.com. 